\title{
THERMOACOUSTIC REFRIGERATION SYSTEMS: RECENT DEVELOPMENTS
}

\author{
B. Farouk ${ }^{1}$ and D. S. Antao ${ }^{2}$ \\ ${ }^{1}$ Department of Mechanical Engineering, Drexel University, Philadelphia., PA 19104 U.S.A. \\ . *Corresponding author's e-mail: bfarouk@coe.drexel.edu \\ ${ }^{2}$ Department of Mechanical Engineering, Massachusetts Institute of Technology, Cambridge, MA
}

Keywords: Pulse tube, thermoacoustic refrigeration, inertance tube, regenerator phase angle,

\section{Introduction}

In this paper, we first review our recent experimental and computational studies on the orificetype [1] and inertance-type [2] pulse tube refrigerators along with related optimization studies. The experimental studies are done at various values of mean pressure of helium $(\sim 0.35 \mathrm{MPa}$ to $2.2 \mathrm{MPa}$ ), different amplitudes of pressure oscillations, and frequency of operation and size of inertance length and orifice opening. Time-dependent axisymmetric CFD simulations of the inertance-type and orifice-type pulse tube refrigerators are reported where the transient as well as the cycle-averaged operation of the in-line pulse tube refrigerator is studied for the processes occurring in the system. Phase relation between mass flow and pressure waves are also reported for both systems. These relations play important roles in the performance of thermoacoustic devices. While passive forms of phase shifting is considered in [1,2], we also present numerical results for a thermoacoustic refrigerator - where active mechanical phase shifting is achieved via a spring-mass system that replaces the inertance tube and the orifice opening - traditionally used in passive phase shifting systems. In an active phase shifting system (see figure below), greater control of phase shifting is achieved - which would produce lower temperatures at the cold spot.

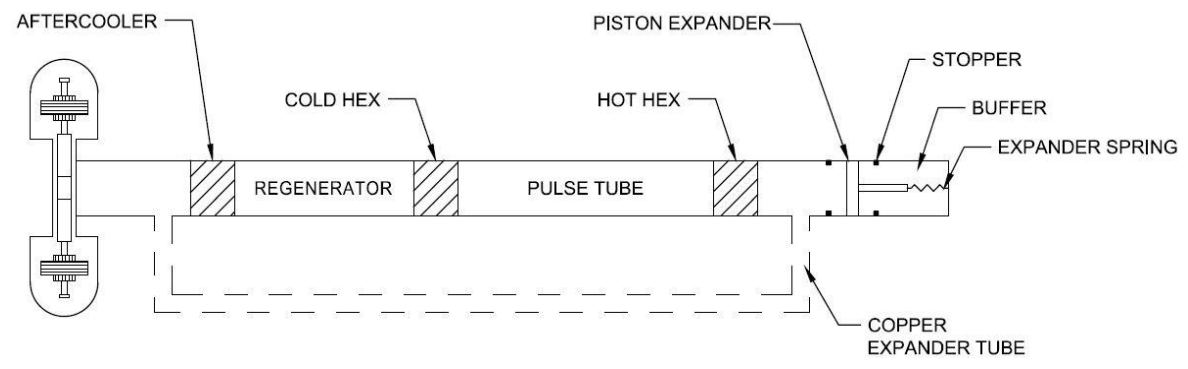

Figure 1: Schematic of an active phase shifting pulse tube refrigerator [3]

\section{Computational model}

The compressible form of the Navier-Stokes equations is considered for the flow simulations of the pulse tube refrigerators. For the porous media regions (i.e. the regenerator and the heat exchangers), we employ the thermal non-equilibrium porous media model. In the nonequilibrium model, the gas and the solid temperatures are different in the porous media. The effect of heat transfer between the gas and the solid phases are considered in the energy equations of the gas and solid phase regions. The cycle-averaged (quasi-steady) and temporal experimental and computational results are also compared. The comparison between the experimental measurements and model predictions are found to improve when the effects of wall thicknesses of the various components of the pulse tube refrigerators are included in the 
CFD model. The computational fluid dynamic model developed has been used to analyze the inertance-type, the orifice-type and the mechanical phase-shifting pulse tube refrigerators with high pressure helium as the working fluid.

\section{Experimental system}

An orifice-type pulse tube refrigerator was designed and built. The pressure wave generator used in the experiments was a Q-Drive twin-STAR linear motor. The linear motor has a swept volume of 15.55 cubic centimeters and a maximum operating pressure of $2.5 \mathrm{MPa}$. The heat exchangers were made from copper tubing (due to its high thermal conductivity) and the other components of the refrigerator were made of 316-type stainless steel tubing (stainless steel exhibits relatively low thermal conductivity and high strength in the temperature range of 350 - .100 K). The various components were connected by flange couplings. The flanges were welded on to the component tubing. Indium wire O-rings were found to provide the best seal for the system operating with high pressure helium and large temperature gradients. The orifice used in the system was a NOSHOK brass needle valve (101-MMB) capable of a maximum flow coefficient of 0.42 . The aftercooler, cold and hot heat-exchanger tubing were filled with stacked copper woven square-mesh screen. The mesh screen in the heat exchangers acts as the primary heat-exchanger between the gas refrigerant and the tube walls. The regenerator tubing houses the regenerator material which is a high thermal capacity and low thermal conductivity permeable material. The regenerator is composed of stacked stainless steel woven square-mesh screen.

\section{Results and discussion}

Figure 2a shows a typical computed temporal evolution of the gas and solid temperature at the exit of the cold heat exchanger and inlet to the orifice-type pulse tube section. The banded
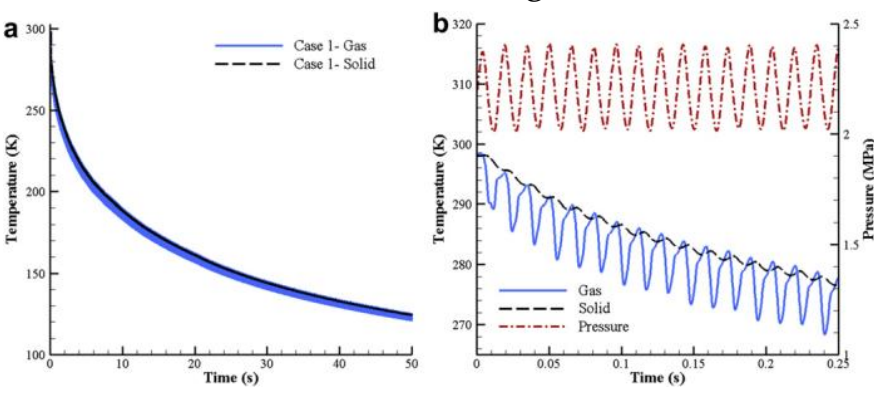

Figure 2. (a) Temporal variation of the gas and solid temperature at the exit of the cold heat-exchanger for the entire simulation, (b) Gas and solid temperature and pressure profiles at the exit of the cold heat-exchanger at the start of the simulations

profiles are cyclic variation of the gas temperature in the system. This cyclic nature of both the gas and solid temperature is highlighted in Figure $2 b$. The gas temperature decreases rapidly at the start; however the decrease in temperature is exponential over time. The solid temperature oscillates too, however the oscillations have much lower amplitude as compared to the gas temperature.

Computational results for all three systems considered are presented in the paper while only experimental results are presented for the orifice-type pulse tube refrigerator

\section{Acknowledgements}

The authors would like to thank Dr. Ray Radebaugh for his continued interest in our work

\section{References}

[1] D.S. Antao, B. Farouk, Experimental and numerical investigations of an orifice type cryogenic pulse tube refrigerator, Applied Thermal Engineering 50 (2013) 112e123, 50 (2013) 112-123.

[2] D.S. Antao, B. Farouk, Numerical and experimental characterization of the inertance effect on pulse tube refrigerator performance, International Journal of Heat and Mass Transfer, 76 (2014) 33-44.

[3] R. Radebaugh, Cryocoolers: the state of the art and recent developments, Journal of Physics: Condensed Matter, 21(16) (2009) 164219 (164219 pp.). 